\title{
CAMBIOS EN LA DEMANDA POR PROGRAMAS PROFESIONALES DE PREGRADO Y EL DESAFÍO QUE ELLO IMPONE A LAS POLÍTICAS PÚBLICAS Y A LA UNIVERSIDAD
}

En los últimos 25 años se produjo una profunda transformación de la educación superior en Chile. Se instaló un sistema que, a diferencia de décadas anteriores, combina la oferta pública con una oferta privada creciente. Después de la reforma de 1980/81 se produjo una apertura del sistema que generó la creación de universidades privadas y también de sedes regionales de las universidades tradicionales, a lo que se agregó la creación de dos nuevos tipos de instituciones de educación superior: los institutos profesionales y los centros de formación técnica.

En la actualidad existen en el país 25 universidades tradicionales y derivadas agrupadas en el Consejo de Rectores de las Universidades Chilenas, 37 universidades privadas, 45 institutos profesionales y 115 centros de formación técnica. Esto ha llevado a una fuerte expansión del sistema, con 600.000 estudiantes en educación superior en el año 2005, cifra que representa un aumento mayor al $100 \%$ en relación con lo que ocurría hace tan sólo 15 años, y que se estima continuará aumentando a cerca de 800.000 alumnos hacia el año 2010.

Por otra parte, en Chile se está produciendo un aumento de la población en edad de asistir a la educación superior, al punto que dos de cada tres jóvenes en edad de egreso de la enseñanza media completan sus estudios en este nivel, e ingresan a la educación universitaria una de cada dos personas jóvenes. La cobertura actual en el segmento de edad entre 18 y 24 años es de 38\%. Cabe destacar que en 1965 era de sólo el 6\%, y en 1985 de un 16\%. A un ritmo de crecimiento de un 6\% anual, se espera que en el año 2010 la cobertura sea de un $42 \%$ en este grupo de edad. 
Este incremento significa que, en términos de posibilidades de acceso a la educación superior, Chile ha dejado de ser un sistema de elite, llegando a constituir uno masivo, de manera similar a lo que han experimentado la mayoría de los países de ingresos medios y altos. Esta realidad significa, por cierto, grandes desafíos. Entre otros, si se considera que la cobertura de educación superior en los quintiles más bajos se triplica, responder de manera eficiente y eficaz a las crecientes demandas de créditos y becas para los jóvenes de escasos recursos.

En 2004, de una matrícula total de pregrado de 594.247 alumnos, un $17 \%$ correspondía a carreras cortas de carácter técnico y un $83 \%$ a universitarias. A su vez, la matrícula en las universidades del Consejo de Rectores fue de 246.286 alumnos (58\%) y la de las privadas fue de 177.271 alumnos (42\%). Cabe destacar que la matrícula universitaria ha aumentado más de tres veces desde 1983 a la fecha, y que la participación de las instituciones privadas en este crecimiento es bastante significativa: en 1983 contaban con una matrícula inferior a 3.000 alumnos y en 2005 ésta alcanza a más de 175.000 estudiantes.

Por otra parte, se ha producido una importante expansión geográfico-territorial. En la actualidad -en todo el país, aunque la mayor concentración se sigue observando en la Región Metropolitana- hay 131 sedes de las universidades del Consejo de Rectores y 107 que corresponden a 37 universidades privadas. Quienes más han contribuido a esta expansión han sido las 17 universidades derivadas, con un promedio de 5,94 sedes por cada institución, contra 3,75 correspondientes a las ocho universidades tradicionales, y 2,89 a las privadas.

Dentro del Consejo de Rectores de las Universidades Chilenas, diez universidades concentran más del $60 \%$ de la oferta de pregrado, siendo las principales la Universidad de Chile (con un 10,3\% de la oferta), la Universidad de Santiago y la Universidad de Concepción. En conjunto con las universidades privadas, estas 
diez reúnen casi un $40 \%$ del total de la matrícula nacional. Diez de las privadas concentran el $67 \%$ de la matrícula de pregrado y participan con poco más del $25 \%$ de la matrícula total de esta clase de oferta.

Las universidades también se diferencian de acuerdo con su tamaño, si reciben aporte directo del Estado y en cuanto a su selectividad. Sobre este último criterio, sólo las universidades del Consejo de Rectores exigen como requisito, además de las notas de enseñanza media, alcanzar en la Prueba de Selección Universitaria (PSU) un puntaje mínimo, que es variable, y que en el caso de las universidades más selectivas es mayor que 600 puntos.

Según la última guía INDICES ${ }^{1}$, publicada por el Consejo Superior de Educación (CSE), existen actualmente más de 5.000 alternativas de estudio en educación postsecundaria. Cabe preguntarse, ¿cuáles son las carreras que más han crecido?

Entre 1983 y 2003, en todo el sistema, las carreras tecnológicas, de ciencias sociales y de administración y comercio absorben dos tercios de la expansión del mercado. Las de menor contribución porcentual son las del área de humanidades, que incluso experimentan un retroceso durante este periodo, junto con las de ciencias básicas y agropecuaria.

En el caso de las universidades públicas, el incremento de la matrícula se produce principalmente en las tecnologías y en educación, que significa un poco menos de la mitad del aumento (de 105.000 alumnos en 1983 a 230.000 en 2003). Siguen en importancia las carreras de salud, agropecuaria, arte, arquitectura y derecho. En el caso de las privadas, que agregan 145.000 alumnos durante ese periodo, la mayor proporción del aumento corresponde a las áreas de ciencias sociales y tecnologías ( $50 \%$ del total). Siguen en importancia educación, derecho, arte y arquitectura.

1 Indicadores, números y datos sobre instituciones y carreras de educación superior (INDICES). 
En la actualidad, el 20\% de la matrícula se concentra en sólo diez carreras: Pedagogía General Básica, Contador Auditor, Administración de Empresas, Derecho, Trabajo Social, Psicología, Educación Parvularia, Ingeniería Comercial, Periodismo y Medicina Veterinaria. A modo de ejemplo, baste señalar que existen 132 opciones para la carrera de Contador Auditor, 130 para Administración de Empresas, 132 para Pedagogía General Básica, 109 para Psicología, 120 para Derecho y 101 en Ingeniería Comercial.

Según lo que informa el Observatorio del $\mathrm{Empleo}^{2}$, la reserva actual de profesionales del país alcanza a 540.000 personas, de las cuales un 44\% tiene menos de 35 años. Más del 40\% de los profesionales egresó de la universidad después de 1995 y, en algunas carreras, el $80 \%$ se ha titulado en los últimos diez años. El 88\% de quienes completan la educación superior está empleado, mientras que, de las personas que tienen estudios superiores incompletos, sólo lo está un $47 \%$. Un profesional universitario gana en Chile 3,68 veces más de lo que gana una persona con educación secundaria completa.

Esta es la realidad actual del sistema de educación superior en Chile. Habría que indicar, sin embargo, que el sistema presenta un conjunto de debilidades que, sin duda, merecen una reflexión.

Lo primero que habría que destacar es la amplia oferta del sistema. Hoy las posibilidades de ingreso a la educación superior se han ampliado de manera tal, que en algunas universidades -particularmente en las privadas, aunque también en algunas públicas- los cupos ofrecidos en las carreras de pregrado no se completan. El año 2003, en todo el sistema universitario, quedaron sin cubrir 4.612 vacantes, de las cuales el $87,2 \%$ correspondió a universidades privadas. Esto podría deberse, a lo menos, a dos factores: a que las carreras que enfrentan esta situación son poco atractivas, entre otras razones por saturación de mercado,

2 Véase http://www.futurolaboral.cl 
o a que no existe un sistema eficiente de becas y préstamos que permita a estudiantes de escasos recursos acceder a ellas.

Lo segundo que importa es preguntarse si la expansión que ha experimentado la oferta de carreras de pregrado, tanto del sistema público como del privado, responde adecuadamente a las necesidades reales de profesionales y técnicos del país, en la perspectiva de alcanzar un desarrollo y crecimiento adecuados en los ámbitos económico, político, social y cultural, y que beneficie a las personas.

A la luz de los antecedentes de que se dispone esto no es así, ya que la institucionalidad educacional vigente no cuenta con atribuciones que permitan regular el crecimiento irracional que se ha dado en la creación de universidades privadas y, por lo tanto, de oferta de carreras de pregrado, que en no pocos casos responde más al afán de obtener un beneficio económico que a las necesidades reales del país.

En tercer lugar, observamos una desigual calidad de la oferta a los estudiantes. En muchos casos las carreras que se ofrecen distan mucho de los estándares que se requieren, esto sin mencionar los casos de algunas universidades que, una vez matriculados sus estudiantes, han quebrado por insolvencia económica, dejando a los jóvenes sin posibilidad alguna de exigir el cumplimiento de un contrato por la entrega de "un servicio educacional".

En este sentido, la acreditación de las instituciones de educación superior y de las carreras de pregrado es una herramienta que debiera permitir a los postulantes conocer de modo transparente cuál es la universidad que les ofrece y asegura la mejor formación profesional. Actualmente 18 universidades han acreditado su calidad; pero, en el futuro, todas tendrán que pasar por dicho proceso. En el caso de la acreditación de carreras, de 500 carreras que participan en este proceso 180 han obtenido ya su acreditación. Esta herramienta debe permitir una mejor selección de las universidades de acuerdo con su calidad. 
Lo cuarto es que la oferta de carreras de pregrado de las universidades no siempre está en relación con la posibilidad real de encontrar un empleo en el futuro. Si bien obtener un título profesional favorece la posibilidad de encontrarlo, esto no siempre significa que se puede obtener uno de calidad, como tampoco asegura hallarlo en el ámbito profesional en el cual se obtuvo el título profesional.

En este sentido, se dan diversas situaciones. Por una parte, está el profesional que encuentra trabajo de acuerdo con sus habilidades y competencias y con un nivel de remuneración aceptable; por otra, aquel que trabaja en puestos de menor jerarquía en el ámbito de su competencia y, por lo tanto, su remuneración no es la que corresponde a su título universitario o a las expectativas que tenía. Ejemplos hay muchos: ingenieros comerciales que trabajan como cajeros de banco, periodistas que trabajan como relacionadores públicos o abogados que trabajan en tareas menores en la judicatura.

También están los casos, y no son pocos, de aquellos profesionales que, una vez titulados, se ven obligados a aceptar puestos de trabajo que no tienen que ver con sus habilidades y competencias profesionales. Por cierto que, en estos casos, la remuneración a la cual acceden es inferior a la que podrían obtener si ejercieran profesionalmente, pero es mayor a que podrían lograr si no contaran con una profesión universitaria. Esta es una distorsión tremenda entre la oferta de carreras de pregrado y la demanda de las mismas, lo que, sin duda, se debe corregir de modo urgente.

Como conclusión, es necesario mencionar las diferentes políticas públicas comprometidas actualmente en el sistema de educación superior, que tanto las autoridades políticas como las universitarias debieran enfrentar a la brevedad.

Primero, establecer una política coherente y consistente de becas y crédito universitario, que apunte, en lo fundamental, a 
entregar oportunidades a todos los sectores, independientemente de otra condición que no sea el mérito académico. Al respecto, el país debiera definir una política que asegure la formación de las nuevas generaciones para su ingreso a la sociedad del conocimiento.

Segundo, regular el crecimiento irracional que ha tenido hasta ahora el sistema de educación superior. Esta política debiera apuntar principalmente a regular la oferta de carreras de pregrado, que en el ámbito de las universidades privadas se rige sólo por el mercado, sin considerar los requerimientos de desarrollo del país.

Tercero, otra de las políticas públicas se refiere a la transparencia de la información para quienes aspiran a ingresar a la educación superior. En este sentido, se hace necesario vigorizar la herramienta de la acreditación de las carreras de pregrado que ofrecen tanto las universidades públicas como privadas. Sería conveniente y necesario, para un proceso de toma de decisiones realmente informado, que éstas dieran a conocer sus carreras acreditadas, aquellas en proceso de acreditación y también las que no fueron acreditadas.

En cuarto lugar, y finalmente, se requiere que el Estado regule no sólo el crecimiento explosivo e irracional de creación de universidades sino, también, el tipo de carreras de pregrado que las diferentes universidades ofrecen, las que, sin duda, debieran estar en correspondencia con los requerimientos del país, sobre todo porque con ello estaríamos asegurando el campo laboral a nuestros egresados. En este sentido, las universidades públicas de mayor complejidad podrían jugar un rol fundamental, dado su tamaño e incidencia en el mercado.

No cabe duda de que el sistema de educación superior enfrenta una situación delicada, un punto de inflexión que es necesario y urgente enfrentar. Condición necesaria para ello es que los diferentes actores nos pongamos a trabajar desde ya. Pero se requiere también que el gobierno, en el marco de sus atribuciones, asuma un rol más activo en la racionalización del sistema. 


\section{Referencias bibliográficas}

Armanet, P., Formación Universitaria para el siglo XXI, En Foco, 44, Serie Expansiva, disponible en www.expansiva.cl

Armanet, P. y Uribe, D., Escenarios futuros de la Educación Superior Chilena: Desafíos de medición, Presentación en Seminario Internacional "Midiendo las Desigualdades", del Instituto Nacional de Estadísticas, 31 de mayo de 2005.

Bernasconi, A. y Rojas, F., Informe sobre la Educación Superior en Chile: 1980-2003.

Brunner, J.J., Elacqua, G., Tillet, A. y col., Guiar El Mercado. Informe Sobre la Educación Superior en Chile, marzo 2005, disponible en http:// www.uai.cl

Comisión Nacional de Acreditación de Programas de Pregrado, disponible en www.cnap.cl

Consejo Superior de Educación, disponible en www.cse.cl

Estructura del Mercado de la Educación Superior, Resumen Ejecutivo, disponible en www.gemines.cl 